\title{
Polymorphism of HDAC9 Gene Is Associated with Increased Risk of Acute Coronary Syndrome in Chinese Han Population
}

\author{
Zhenhua Han, ${ }^{1}$ Xin Dong, ${ }^{1}$ Chaoying Zhang, ${ }^{1}$ Yue Wu, ${ }^{2}$ Zuyi Yuan, ${ }^{2}$ and Xinhong Wang ${ }^{1}$ \\ ${ }^{1}$ Department of Cardiology Medicine, The Second Affiliated Hospital of Xi'an Jiaotong University, Xi'an, Shaanxi 710004, China \\ ${ }^{2}$ Department of Cardiovascular Medicine, First Affiliated Hospital of Medical College and Key Laboratory of Environment \\ and Genes Related to Diseases, Xian Jiaotong University, Ministry of Education, Xian, Shaanxi, China
}

Correspondence should be addressed to Xinhong Wang; wangxinhongshx@163.com

Received 18 April 2016; Accepted 20 July 2016

Academic Editor: Gelin Xu

Copyright (C) 2016 Zhenhua Han et al. This is an open access article distributed under the Creative Commons Attribution License, which permits unrestricted use, distribution, and reproduction in any medium, provided the original work is properly cited.

\begin{abstract}
Recent genome-wide association studies (GWAS) have indicated an association of histone deacetylase 9 (HDAC9) genetic variant with large-vessel stroke and coronary artery disease, among the European population. However, whether HDAC9 gene is associated with an increased susceptibility to acute coronary syndrome (ACS) in Chinese Han population is not known. A total of 472 patients, including patients with ACS $(N=309)$, and those with chest pain syndrome (controls, $N=163$ ) were enrolled. Genotyping for HDAC9 gene was performed using the ligation detection reaction assay. A series of statistical analyses were performed to investigate the correlation between HDAC9 gene SNPs and the susceptibility to ACS. The results revealed a significant association of rs 2240419 with ACS risk in which the A allele $(P=0.047)$ and the A allele carriers (AA + AG) $(P=0.037)$ were more likely to be in ACS group as compared to those in the control group. None of two other SNPs, rs2389995 and rs2107595, were significantly associated with ACS risk $(P>0.05)$. Logistic regression analyses further revealed an increased risk for ACS in A allele carrier among rs2240419 genotypes, as compared to those with GG homozygotes (odds ratio: 1.869, 95\% CI 1.143, 3.056, $P=0.013$ ). A significant correlation between rs2240419 polymorphism of HDAC9 gene and the susceptibility to ACS in Chinese Han population was observed in this study.
\end{abstract}

\section{Introduction}

Acute coronary syndrome (ACS), which includes ST-segment elevation myocardial infarction (STEMI), non-ST-segment elevation myocardial infarction (NSTEMI), and unstable angina (UA), is a major cause of morbidity and mortality in the western world. The common denominator of ACS is a pathophysiologic process characterized by rupture of an atherosclerotic plaque, altered coronary vasomotor tone, platelet aggregation, and thrombosis. Compelling evidence from twin and epidemiological studies suggests a genetic basis underlying ACS $[1,2]$.

Histone deacetylases (HDACs) are a family of enzymes which balance the acetylating activities of histone acetyltransferases on chromatin remodeling and play essential roles in regulating gene transcription $[3,4]$. About 18 mammalian HDACs have been identified and grouped into four classes based on their structural and biochemical characteristics [5]. HDAC9 is expressed in heart, T lymphocytes, endothelium, adipose tissues, vascular smooth muscle cells, and macrophages [5-7]. A recent genome-wide association study (GWAS) linked a variant of HDAC9 (rs11984041) on chromosome 7p21.1 with large-vessel ischemic stroke in individuals with European ancestry [8]. Han et al. reported that two SNPs, rs2389995 and rs2240419 on HDAC9, were significantly associated with large-vessel ischemic stroke risk in the Chinese population, although rs11984041 was not polymorphic in the Chinese population [9]. Upregulation of HDAC9 expression was observed in human atherosclerotic plaques including in carotid, aortic, and femoral plaques, which appears to implicate the altered HDAC9 expression in promoting atherosclerosis $[6,10]$. Cao et al. showed that macrophage HDAC9 upregulation is atherogenic via suppression of cholesterol efflux and generation of 


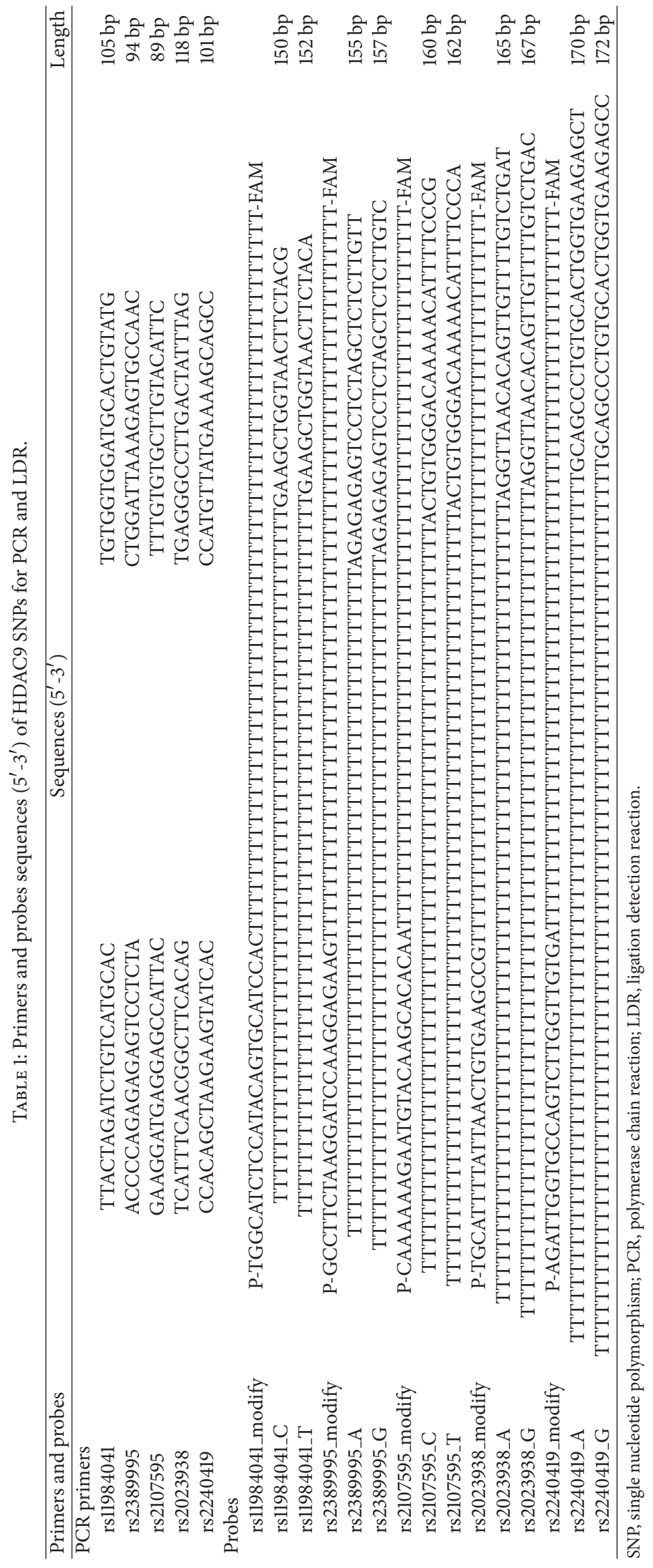


TABLE 2: Clinical characteristics of subjects by study group.

\begin{tabular}{|c|c|c|c|}
\hline Characteristics & Control $(N=163)$ & $\operatorname{ACS}(N=309)$ & $P$ \\
\hline $\operatorname{Sex}(m / f)$ & $94 / 69$ & $229 / 80$ & $<0.001$ \\
\hline Age (years) & $59.66 \pm 10.82$ & $60.61 \pm 11.98$ & 0.401 \\
\hline Hypertension (\%) & 54.6 & 57.9 & 0.488 \\
\hline Diabetes (\%) & 13.1 & 25.2 & 0.002 \\
\hline Smoking (\%) & 29.4 & 55 & $<0.001$ \\
\hline SBP (mmHg) & $127.54 \pm 22.74$ & $127.74 \pm 22.84$ & 0.927 \\
\hline DBP (mmHg) & $78.82 \pm 13.55$ & $78.83 \pm 13.89$ & 0.998 \\
\hline HR (bpm) & $71.60 \pm 14.64$ & $74.72 \pm 14.56$ & 0.028 \\
\hline $\operatorname{LVEF}(\%)$ & $63.45 \pm 12.19$ & $59.05 \pm 12.16$ & $<0.001$ \\
\hline hs-CRP (mg/L) & $2.23 \pm 3.07$ & $4.34 \pm 4.26$ & $<0.001$ \\
\hline FBS (mmol/L) & $5.88 \pm 1.98$ & $7.10 \pm 3.43$ & $<0.001$ \\
\hline $\mathrm{TC}(\mathrm{mmol} / \mathrm{L})$ & $3.90 \pm 0.91$ & $3.85 \pm 0.96$ & 0.556 \\
\hline TG (mmol/L) & $1.60 \pm 1.21$ & $1.72 \pm 1.18$ & 0.302 \\
\hline $\mathrm{HDL}-\mathrm{C}(\mathrm{mmol} / \mathrm{L})$ & $0.98 \pm 0.24$ & $0.94 \pm 0.63$ & 0.484 \\
\hline $\mathrm{LDL}-\mathrm{C}(\mathrm{mmol} / \mathrm{L})$ & $2.32 \pm 0.78$ & $2.28 \pm 0.85$ & 0.617 \\
\hline ApoA1 (g/L) & $1.14 \pm 0.21$ & $1.07 \pm 0.19$ & $<0.001$ \\
\hline ApoB (g/L) & $0.76 \pm 0.21$ & $0.77 \pm 0.22$ & 0.752 \\
\hline Statins (\%) & 83.4 & 98.0 & $<0.001$ \\
\hline ACEI/ARB (\%) & 69.9 & 88.2 & $<0.001$ \\
\hline$\beta$-blocker (\%) & 62.0 & 86.6 & $<0.001$ \\
\hline CCB $(\%)$ & 24.5 & 20.3 & 0.293 \\
\hline
\end{tabular}

ACS, acute coronary syndrome; SBP, systolic blood pressure; DBP, diastolic blood pressure; HR, heart rate; LVEF, left ventricular ejection fraction; hsCRP, high sensitivity C-reactive protein; FBS, fasting blood sugar; TC, total cholesterol; TG, triglycerides; HDL-C, high-density lipoprotein cholesterol; LDL-C, low-density lipoprotein cholesterol; ApoAl, apolipoprotein A1; ApoB, apolipoprotein B; ACEI, angiotensin converting enzyme inhibition; $\mathrm{ARB}$, angiotensin receptor blocker; $\mathrm{CCB}$, calcium channel blocker.

alternatively activated macrophages in atherosclerosis [7]. Moreover, HDAC9 is also a common genetic variant that shares genetic susceptibility to ischemic stroke and coronary artery disease (CAD) [11].

In the present study, we aimed to examine the association between five SNPs of the HDAC9 gene and risk of ACS in a Chinese population. Our study represents one of the first efforts to associate risk alleles in HDAC9 gene region with ACS in the Chinese population.

\section{Results}

2.1. Clinical Characteristics. Baseline characteristics for all patients are shown in Table 2. Proportion of males, number of smokers, incidence of diabetes, heart rate, fasting blood glucose, and hs-CRP were higher in ACS group as compared to those in the control group. Left ventricular ejection fraction (LVEF) and ApoA1 level were significantly lower in patients with ACS as compared to those in the controls. No other significant intergroup differences were observed.

2.2. Association of Allele and Genotypic Frequency Distribution with Susceptibility of ACS. Two SNPs, rs11984041 and rs2023938, were found to be nonpolymorphic in the Chinese population because rare five-T allele carrier of SNP
TABLE 3: Genotypes and alleles distribution of HDAC9 gene SNPs in ACS and control.

\begin{tabular}{cccccc}
\hline \multicolumn{4}{c}{ Control } & \multicolumn{2}{c}{ ACS } \\
& $N$ & $\%$ & $N$ & $\%$ & \\
\hline rs2389995 & & & & & \\
Genotypes & & & & & \\
AA & 113 & 69.30 & 198 & 64.10 & \\
AG & 45 & 27.60 & 104 & 33.70 & 0.377 \\
GG & 5 & 3.10 & 7 & 2.30 & \\
Allele & & & & & \\
A & 271 & 83.10 & 500 & 80.90 & 0.401 \\
G & 55 & 16.90 & 118 & 19.10 & \\
rs2107595 & & & & & \\
Genotypes & & & & & \\
CC & 78 & 47.90 & 139 & 45.00 & \\
CT & 73 & 44.80 & 130 & 42.10 & 0.183 \\
TT & 12 & 7.40 & 40 & 12.90 & \\
Allele & & & & & \\
C & 229 & 70.20 & 408 & 66.00 & 0.188 \\
T & 97 & 29.80 & 210 & 34.00 & \\
rs2240419 & & & & & \\
Genotypes & & & & & \\
AA & 9 & 5.50 & 18 & 5.80 & \\
AG & 48 & 29.40 & 127 & 41.10 & $0.037^{*}$ \\
GG & 106 & 65.00 & 164 & 53.10 & \\
Allele & & & & & \\
A & 66 & 20.20 & 163 & 26.40 & $0.047^{*}$ \\
G & 260 & 79.80 & 455 & 73.60 & \\
\hline
\end{tabular}

ACS, acute coronary syndrome; $*$ denotes $P<0.05$.

rs11984041 and five-G allele carrier of SNP rs2023938 were found among both patients with ACS and controls. Genotype distributions for other three SNPs were in Hardy-Weinberg equilibrium in both groups $(P>0.05$, data not shown). Table 3 shows the association of HDAC9 gene alleles or genotypes with ACS.

We compared allele and genotype frequencies of the three SNPs between the two groups. We observed an association of rs2240419 with ACS risk, in which the A allele $(P=0.047)$ and the A allele carriers $(\mathrm{AA}+\mathrm{AG})(P=0.037)$ were more frequent in ACS group, in contrast to those in the control group, whereas, none of the two other SNPs, rs2389995 and rs2107595, were significantly associated with ACS risk $(P>$ 0.05).

Multivariate logistic regression analysis of SNP rs2240419, adjusted for differences in age, sex, cigarette smoking, hypertension, diabetes, fasting blood glucose, hs-CRP, and lipoprotein variables, confirmed the independent association (Table 4). A allele carriers in rs2240419 genotypes had an increased ACS risk as compared to that associated with the GG homozygotes; the adjusted OR was 1.869 (95\% CI 1.143, $3.056, P=0.013$ ). Diabetes, smoking, and hs-CRP were the other variables that independently contributed to the risk of ACS, the adjusted ORs being 2.306 (95\% CI 1.232, 4.317, $P=$ 0.009 ) for diabetes, 3.909 (95\% CI 2.142, 7.134, $P<0.001)$ for smoking, and 1.126 (95\% CI 1.043, 1.216, $P=0.002)$ for hsCRP. 
TABLE 4: Results of logistic regression analysis showing risk factors for ACS.

\begin{tabular}{lcc}
\hline Covariates & Odds ratio $(95 \% \mathrm{CI})$ & $P$ \\
\hline rs2240419 & $1.869(1.143,3.056)$ & 0.013 \\
Sex & $0.923(0.516,1.653)$ & 0.788 \\
Age & $1.018(0.996,1.040)$ & 0.112 \\
Hypertension & $1.334(0.827,2.152)$ & 0.238 \\
Diabetes & $2.306(1.232,4.317)$ & 0.009 \\
Smoking & $3.909(2.142,7.134)$ & $<0.001$ \\
TC & $1.646(0.529,5.124)$ & 0.390 \\
TG & $1.112(0.886,1.395)$ & 0.360 \\
HDL-C & $1.432(0.556,3.690)$ & 0.457 \\
LDL-C & $0.753(0.226,2.507)$ & 0.644 \\
ApoA1 (g/L) & $0.267(0.035,2.037)$ & 0.203 \\
ApoB $(g / L)$ & $0.435(0.028,6.742)$ & 0.552 \\
hs-CRP & $1.126(1.043,1.216)$ & 0.002 \\
\hline
\end{tabular}

ACS, acute coronary syndrome; TC, total cholesterol; TG, triglycerides; HDL-C, high-density lipoprotein cholesterol; LDL-C, low-density lipoprotein cholesterol; ApoAl, apolipoprotein A1; ApoB, apolipoprotein B.

TABLE 5: Association of rs2240419 genotypes and clinical characteristics.

\begin{tabular}{lccc}
\hline Covariates & AA + AG & GG & $P$ \\
\hline Sex $(\mathrm{m} / \mathrm{f})$ & $136 / 66$ & $187 / 83$ & 0.655 \\
Age & $59.30 \pm 11.40$ & $61.01 \pm 11.70$ & 0.111 \\
Hypertension & 55.9 & 57.4 & 0.750 \\
Diabetes & 25.0 & 18.0 & 0.068 \\
Smoking & 45.0 & 47.0 & 0.668 \\
TC & $3.82 \pm 0.88$ & $3.90 \pm 0.98$ & 0.356 \\
TG & $1.64 \pm 1.01$ & $1.71 \pm 1.32$ & 0.497 \\
HDL-C & $0.91 \pm 0.23$ & $0.99 \pm 0.67$ & 0.158 \\
LDL-C & $2.26 \pm 0.79$ & $2.32 \pm 0.85$ & 0.469 \\
ApoA1 & $1.08 \pm 0.20$ & $1.10 \pm 0.21$ & 0.354 \\
ApoB & $0.75 \pm 0.21$ & $0.77 \pm 0.22$ & 0.424 \\
hs-CRP & $4.03 \pm 4.40$ & $3.33 \pm 3.72$ & 0.091 \\
\hline
\end{tabular}

TC, total cholesterol; TG, triglycerides; HDL-C, high-density lipoprotein cholesterol; LDL-C, low-density lipoprotein cholesterol; ApoA1, apolipoprotein A1; ApoB, apolipoprotein B.

2.3. Relation between the rs2240419 Genotypes and Clinical Data. We also assessed the association of other clinical variables such as age, sex, cigarette smoking, hypertension, diabetes, fasting blood glucose, hs-CRP, and lipid profile, with rs2240419 genotypes (Table 5). No significant intergroup differences were observed among genetic frequencies in rs2240419 with these clinical parameters.

\section{Discussion}

In this study, we detected a significant correlation between rs2240419 polymorphism of HDAC9 gene and the susceptibility to ACS in a Chinese population, while the other four SNPs showed no such association.

A previous genome-wide association study (GWAS) demonstrated a significant association of SNP rs11984041 of
HDAC9 gene with large-vessel stroke among populations of European ancestry [8]. Another GWAS on stroke in the European population revealed an association of polymorphic loci rs2107595 in HDAC9 gene susceptibility to large-vessel stroke [12]. Dichgans et al. evaluated the shared genetic determination of ischemic stroke and CAD and found that SNP rs2107595 on HDAC9 conferred susceptibility to both ischemic stroke and CAD among Caucasians [11]. Based on the HapMap data, SNP rs11984041 was found to be nonpolymorphic among the Chinese population. Our results are supported by two other studies which showed that SNP rs11984041 was not associated with stroke in a Chinese population, while two other SNPs, rs2389995 and rs2240419 of HDAC9, were significantly associated with large-vessel stroke risk $[9,13]$. A recent study by $\mathrm{Su}$ et al. suggests that HDAC9 variant rs2107595 may not be associated with ischemic stroke risk in Chinese Han population [14]. Consistent with this study, we too found SNP rs11984041 to be nonpolymorphic in the Chinese population, and SNP rs2107595 was not significantly associated with ACS risk. This phenomenon may be attributed to genetic heterogeneity among different ethnic populations.

Eighteen HDACs are encoded by different genes and grouped into four classes on the basis of their structural and biochemical characteristics [15]. HDAC9 is a member of Class IIa HDACs. One of the best characterized mechanisms of action of HDAC9 is its ability to interact with MEF-2 and repress MEF-2 activity, which has been linked to suppression of cardiac hypertrophy $[5,16]$. Moreover, HDAC9 expression was upregulated in human atherosclerotic plaques and was also a common genetic variant that shares the genetic susceptibility to ischemic stroke and coronary artery disease [11].

Cao et al. revealed the underlying molecular mechanisms of HDAC9 leading to increase of atherosclerosis in the in vivo models. HDAC9 deletion resulted in upregulation of lipid homeostatic genes, downregulation of inflammatory genes, and polarization of macrophages toward M2-like phenotype [7]. Recently another study found that HDAC9 regulates oxLDL-induced endothelial cell apoptosis by participating in inflammatory reactions [17]. However, we did not observe a significant association between HDAC9 genetic variant and lipoproteins and inflammatory marker C-reactive protein. Thus, the mechanisms underlying the association of a mutant HDAC9 gene with ACS are still unknown.

\section{Experimental Section}

4.1. Subject Population. A total of 472 consecutive patients (309 patients with ACS and 163 patients with chest pain syndrome as controls) were admitted at the Department of Cardiology, the Second Affiliated Hospital of Medical College of Xian Jiaotong University, between January 2012 and January 2015. All patients underwent elective coronary angiography because of the clinical indication. Two independent interventional cardiologists analyzed the coronary angiography results. The diagnosis of ACS was based on angiography, in accordance with the ACC/AHA guidelines. Subjects with chest pain syndrome were defined as having atypical chest pain without significant angiographic coronary 
stenosis, aortic dissection, and pulmonary embolism. Exclusion criteria included cancer, advanced liver or renal dysfunction, active inflammatory or autoimmune diseases, and hematological diseases. The research protocol was approved by the Ethics Committee at the Second Affiliated Hospital of Xian Jiaotong University. Written informed consent was obtained from all the patients; the study complied with the principles enshrined in the Declaration of Helsinki. Demographic variables, cardiovascular risk factors, and past cardiovascular medical history were recorded for all patients.

4.2. Biochemical Analysis. Fasting blood glucose and serum concentrations of lipoproteins, apolipoproteins, and other biochemical parameters were measured in the clinical Biochemistry Department, at the Second Affiliated Hospital of the Medical College of Xi'an Jiaotong University.

4.3. DNA Extraction and Genotyping. Genomic DNA was extracted from peripheral blood lymphocytes (PBLs) using a commercial blood DNA extraction kit (Tiangen Biotech Co., Ltd., Beijing, China) following the manufacturer's protocol. Guided by the literature search, five SNPs in the HDAC9 gene variants were chosen for this study (SNP1 rs11984041, SNP2 rs2107595, SNP3 rs2023938, SNP4 rs2389995, and SNP5 rs2240419) [6, 8-11, 13, 14]. Genotyping for HDAC9 was performed using the ligation detection reaction (LDR) assay (Shanghai Biowing Applied Biotechnology Company) $[18,19]$. All PCR primers and gene probes used in LDR were designed using Primer 3.0 and Oligo 6.0 software (listed in Table 1). Each set of LDR probes is comprised of one common probe and two discriminating probes for the two types. DNA sequences of the HDAC9 gene were amplified by a multiplex polymerase chain reaction (PCR) method. All PCR reactions were carried out in a final volume of $20 \mu \mathrm{L}$ containing $2 \mu \mathrm{L}$ PCR buffer (1x), $3.0 \mathrm{mmol} / \mathrm{L} \mathrm{MgCl}, 2.0 \mathrm{mmol} / \mathrm{L} \mathrm{dNTP,} 2 \mu \mathrm{L}$ primer mix, $0.2 \mu \mathrm{L}$ Taq Polymerase, and $1 \mu \mathrm{L}$ genomic DNA $(50 \mathrm{ng} / \mu \mathrm{L})$. The amplification procedure was performed with an initial denaturation at $95^{\circ} \mathrm{C}$ for $2 \mathrm{~min}$, followed by 40 cycles of denaturation at $94^{\circ} \mathrm{C}$ for $30 \mathrm{~s}$, annealing at $53^{\circ} \mathrm{C}$ for $90 \mathrm{~s}$, and extension at $65^{\circ} \mathrm{C}$ for $30 \mathrm{~s}$, followed by a final extension at $65^{\circ} \mathrm{C}$ for $10 \mathrm{~min}$ in Gene Amp PCR system 9600 (Norwalk, CT, USA). The LDRs were performed in a final volume of $10 \mu \mathrm{L}$ containing $1 \mu \mathrm{L}$ buffer $(1 \mathrm{x}), 1 \mu \mathrm{L}$ probe $\operatorname{mix}(2 \mathrm{pmol} / \mu \mathrm{L})$, $0.05 \mu \mathrm{L}$ Taq DNA ligase (NEB Biotechnology), and $4 \mu \mathrm{L}$ of multi-PCR product. The LDR included an initial incubation at $95^{\circ} \mathrm{C}$ for $2 \mathrm{~min}$, followed by 40 cycles at $94^{\circ} \mathrm{C}$ for $15 \mathrm{~s}$ and $50^{\circ} \mathrm{C}$ for $25 \mathrm{~s}$. The LDR fluorescent products were analyzed by ABI sequencer 3730 . The quality of genotyping was controlled using blinded blood duplicates.

4.4. Statistical Analysis. All statistical analyses were conducted using SPSS software package, version 18.0 (SPSS Inc., Chicago, IL, USA). Quantitative variables are expressed as means \pm Standard Error (SE) and categorical variables as frequency and percentage. Intergroup differences were assessed by Chi-square test or $t$-test. The genotype distributions for HDAC9 SNPs were tested for Hardy-Weinberg equilibrium. Owing to the small number of patients with AA homozygote in rs2240419 ( $N=18$ and $N=9$ for ACS and control, resp.), AA and AG patients in rs2240419 were combined into a single A allele group (AA + AG) to improve the statistical power. The odds ratio (OR) and 95\% confidence interval (95\% CI) were calculated using binary logistic regression model to access associations between SNP rs2240419 and ACS risk. A 2-tailed $P<0.05$ was considered statistically significant.

\section{Conclusion}

In this study, we demonstrated an association between rs2240419 polymorphism and risk of ACS in a Chinese population. Our findings also attest to the genetic heterogeneity between Chinese and other ethnic groups. However, several potential limitations of our study should be noted. Samples collected from a single center may not be sufficient and the chosen SNPs may not represent the entire HDAC9 gene. Future studies with larger sample size representative of the Chinese population are required to validate our findings.

\section{Competing Interests}

The authors have no other funding and financial relationships or competing interests to disclose.

\section{Acknowledgments}

This study was supported by the Social Development, Scientific and Technological Foundation of Shaanxi Province (2015SF021).

\section{References}

[1] T. M. Morgan, H. M. Krumholz, R. P. Lifton, and J. A. Spertus, "Nonvalidation of reported genetic risk factors for acute coronary syndrome in a large-scale replication study," Journal of the American Medical Association, vol. 297, no. 14, pp. 1551-1561, 2007.

[2] M. T. Scheuner, "Clinical application of genetic risk assessment strategies for coronary artery disease: genotypes, phenotypes, and family history," Primary Care, vol. 31, no. 3, pp. 711-737, 2004.

[3] A. Mai, S. Massa, D. Rotili et al., "Histone deacetylation in epigenetics: an attractive target for anticancer therapy," Medicinal Research Reviews, vol. 25, no. 3, pp. 261-309, 2005.

[4] D.-M. Chuang, Y. Leng, Z. Marinova, H.-J. Kim, and C.-T. Chiu, "Multiple roles of HDAC inhibition in neurodegenerative conditions," Trends in Neurosciences, vol. 32, no. 11, pp. 591-601, 2009.

[5] M. Haberland, R. L. Montgomery, and E. N. Olson, “The many roles of histone deacetylases in development and physiology: implications for disease and therapy," Nature Reviews Genetics, vol. 10, no. 1, pp. 32-42, 2009.

[6] H. S. Markus, K.-M. Mäkelä, S. Bevan et al., "Evidence HDAC9 genetic variant associated with ischemic stroke increases risk via promoting carotid atherosclerosis," Stroke, vol. 44, no. 5, pp. 1220-1225, 2013.

[7] Q. Cao, S. Rong, J. J. Repa, R. S. Clair, J. S. Parks, and N. Mishra, "Histone deacetylase 9 represses cholesterol efflux and alternatively activated macrophages in atherosclerosis development," 
Arteriosclerosis, Thrombosis, and Vascular Biology, vol. 34, no. 9, pp. 1871-1879, 2014.

[8] C. Bellenguez, S. Bevan, A. Gschwendtner et al., "Genome-wide association study identifies a variant in HDAC9 associated with large vessel ischemic stroke," Nature Genetics, vol. 44, no. 3, pp. 328-333, 2012.

[9] Y. Han, W. Sun, L. Wang et al., "HDAC9 gene is associated with stroke risk in a Chinese population," Experimental Biology and Medicine, vol. 238, no. 7, pp. 842-847, 2013.

[10] S. Azghandi, C. Prell, S. W. Van Der Laan et al., "Deficiency of the stroke relevant HDAC9 gene attenuates atherosclerosis in accord with allele-specific effects at 7p21.1," Stroke, vol. 46, no. 1, pp. 197-202, 2015.

[11] M. Dichgans, R. Malik, I. R. König et al., "Shared genetic susceptibility to ischemic stroke and coronary artery disease: a genome-wide analysis of common variants," Stroke, vol. 45, no. 1, pp. 24-36, 2014.

[12] M. Traylor, M. Farrall, E. G. Holliday et al., "Genetic risk factors for ischaemic stroke and its subtypes (the METASTROKE Collaboration): a meta-analysis of genome-wide association studies," The Lancet Neurology, vol. 11, no. 11, pp. 951-962, 2012.

[13] G. Qingxu, Z. Yan, X. Jiannan, and L. Yunlong, "Association between the gene polymorphisms of HDAC9 and the risk of atherosclerosis and ischemic stroke," Pathology and Oncology Research, vol. 22, no. 1, pp. 103-107, 2016.

[14] L. Su, T. Shen, B. Liang et al., "Association of GWAS-supported loci rs2107595 in HDAC9 gene with ischemic stroke in southern Han Chinese," Gene, vol. 570, no. 2, pp. 282-287, 2015.

[15] T. A. McKinsey, "Targeting inflammation in heart failure with histone deacetylase inhibitors," Molecular Medicine, vol. 17, no. 5-6, pp. 434-441, 2011.

[16] C. L. Zhang, T. A. McKinsey, S. Chang, C. L. Antos, J. A. Hill, and E. N. Olson, "Class II histone deacetylases act as signalresponsive repressors of cardiac hypertrophy," Cell, vol. 110, no. 4, pp. 479-488, 2002.

[17] X. Han, X. Han, Z. Wang, J. Shen, and Q. Dong, "HDAC9 regulates ox-LDL-induced endothelial cell apoptosis by participating in inflammatory reactions," Frontiers in Bioscience, vol. 21, no. 5, pp. 907-917, 2016.

[18] Z.-J. Chen, H. Zhao, L. He et al., "Genome-wide association study identifies susceptibility loci for polycystic ovary syndrome on chromosome 2p16.3, 2p21 and 9q33.3," Nature Genetics, vol. 43, no. 1, pp. 55-59, 2011.

[19] L. Deng, R. Huang, Z. Chen, L. Wu, and D.-L. Xu, "A study on polymorphisms of elastin gene in Chinese Han patients with isolated systolic hypertension," American Journal of Hypertension, vol. 22, no. 6, pp. 656-662, 2009. 


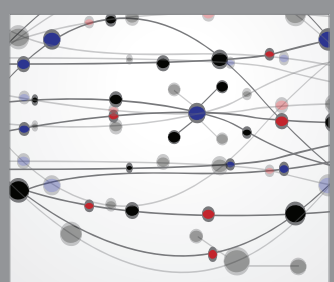

The Scientific World Journal
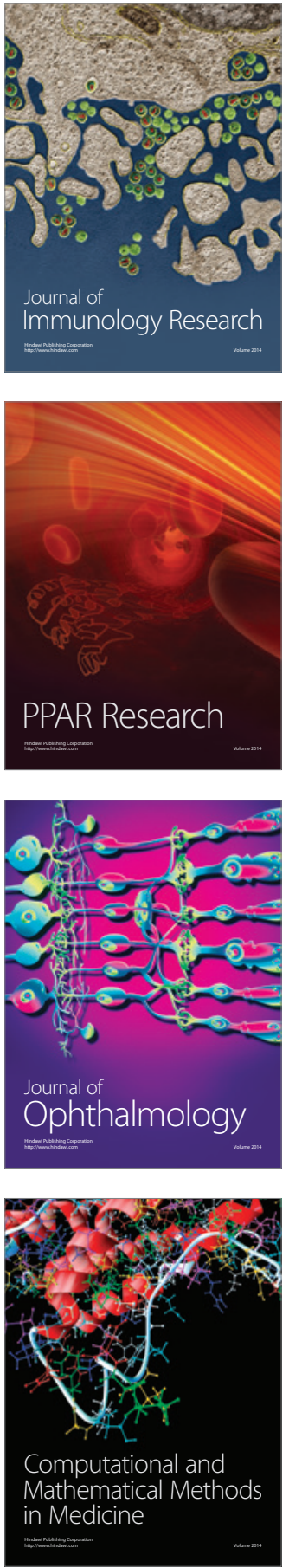

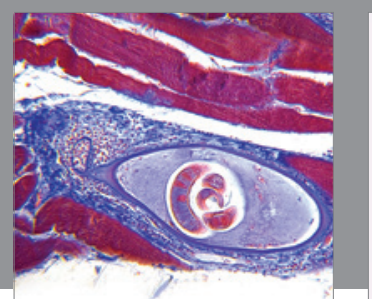

Gastroenterology Research and Practice

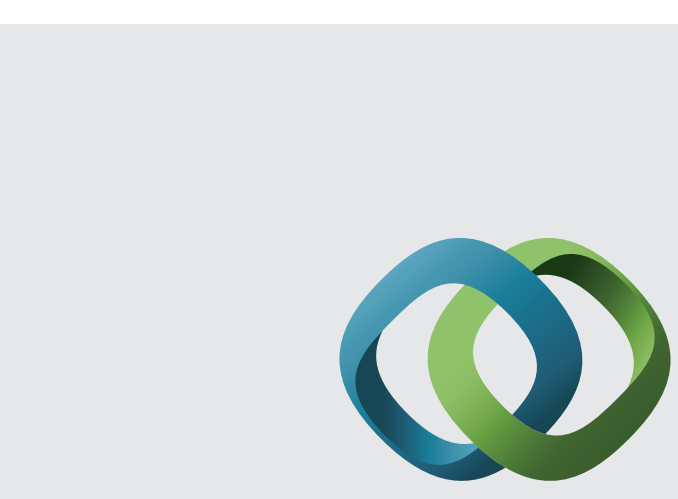

\section{Hindawi}

Submit your manuscripts at

http://www.hindawi.com
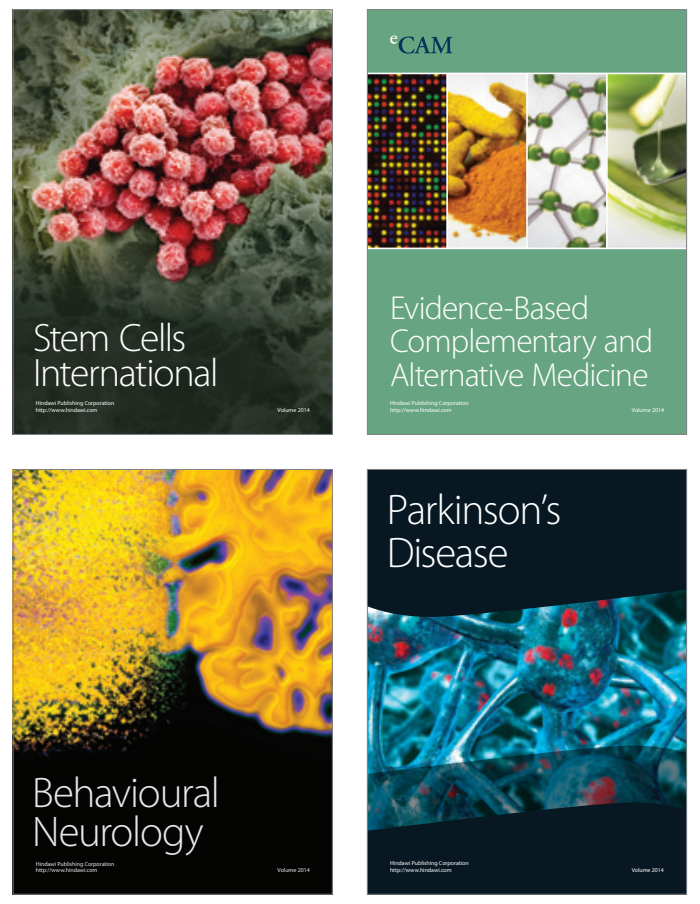
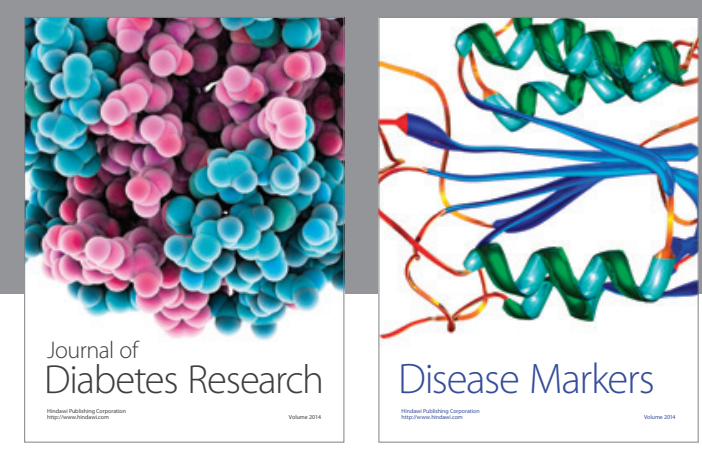

Disease Markers
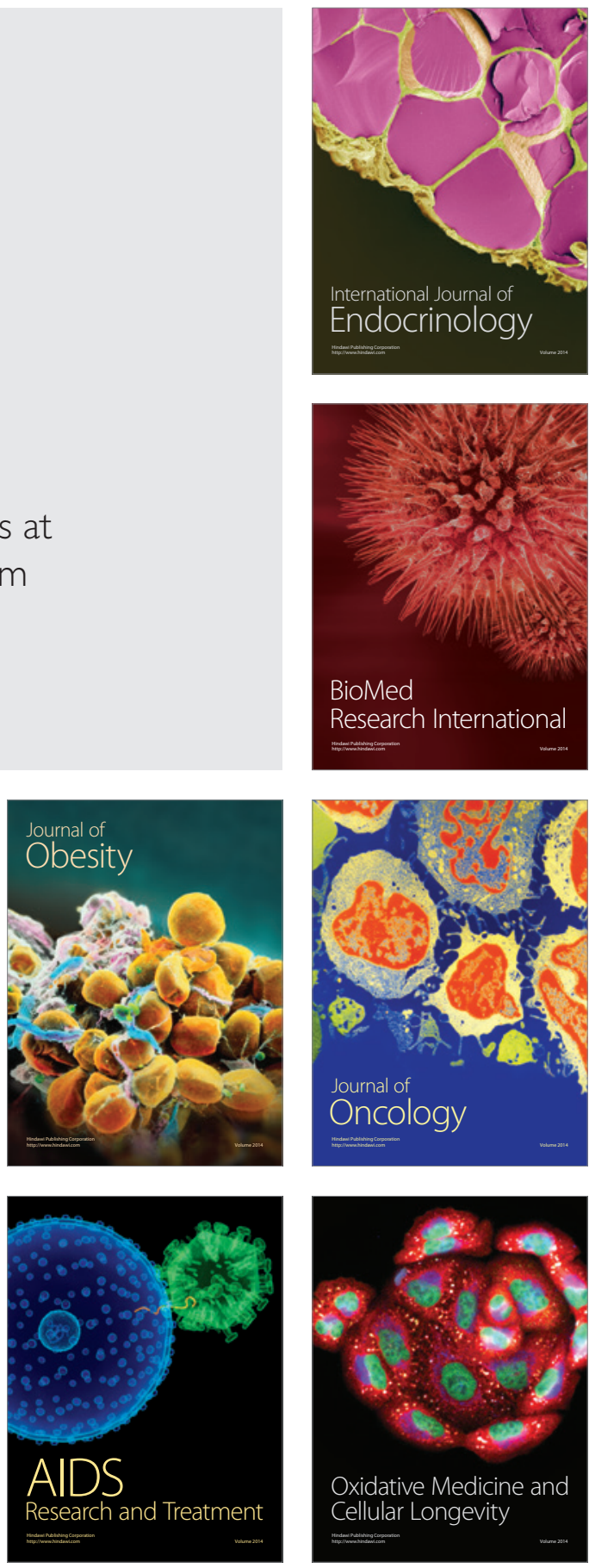